\title{
A SURVEY: TO HARNESS AN EFFICIENT ENERGY IN CLOUD COMPUTING
}

\author{
Malathi. $\mathrm{P}^{1}$, Arumugam. $\mathrm{S}^{2}$ \\ ${ }^{1}$ M.E.Scholar, Department of Computer Science \& Engineering, Nandha Engineering \\ College, Erode-638052, Tamil Nadu, India \\ ${ }^{2}$ Professor, Department of Computer Science \& Engineering, Nandha Engineering \\ College, Erode-638052, Tamil Nadu, India
}

\begin{abstract}
Cloud computing affords huge potential for dynamism, flexibility and cost-effective IT operations. Cloud computing requires many tasks to be executed by the provided resources to achieve good performance, shortest response time and high utilization of resources. To achieve these challenges there is a need to develop a new energy aware scheduling algorithm that outperform appropriate allocation map of task to optimize energy consumption. This study accomplished with all the existing techniques mainly focus on reducing energy consumption.
\end{abstract}

\section{KEYWORDS}

Cloud computing, Energy consumption, Virtualization, renewable energy, Virtual machine

\section{INTRODUCTION}

With the development of high speed networks, there is an alarming rise in its usage comprised of Web queries a day and thousands of e-commerce transactions. A large scale data centers handle this ever increasing demand by consolidating hundreds and thousands of servers with other infrastructure such as cooling, network systems and storage. The development of this commercialization is named as cloud computing. Clouds are sky rocketing virtualized data centers and applications offered as services on a subscription basis. The characteristics exhibited by Clouds are shown in Figure 1

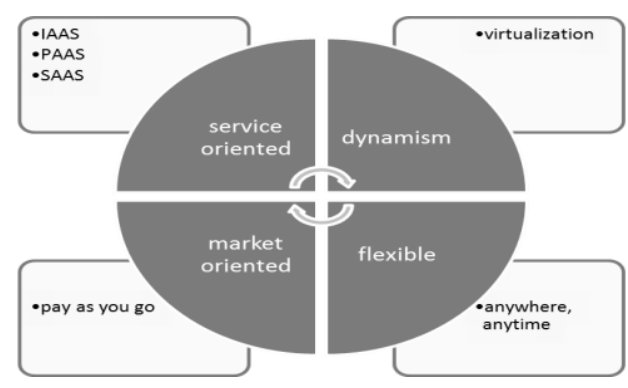

Figure 1.characteristics of cloud computing.

In recent years, a great amount of research on cloud computing to offer low power systems, since serious issue on the sustainability of current technologies and practices. To address the various issues such as resource management in both software and hardware levels to reduce energy consumption. Overload and under load of system is also one of the phenomenon for energy loss 
[5]. According to [15], the recent studies reveal that the ideal system consumes $70 \%$ of energy. The virtualization technology is to resolve those problems [4]. The performance optimization, energy saving, load balancing and on demand resource allocation can be achieved by using good resource management scheme [21]. There is a relationship between energy consumption and resource utilization in cloud computing. If the energy consumption increases tremendously which directly affect the performance of the cloud in a while increases the cost.

In this survey paper, energy harnessing techniques are discussed. The remaining paper is organized as follows: Section 2 presents overview, Section 3 presents the literature review of the existing methods of energy efficient techniques, and Section 4 presents conclusions.

\section{OVERVIEW}

\subsection{Energy Consumption Problem}

The major problem in cloud is energy sprawl. This is due to two reasons. One is manual faults like improper scheduling and work overload $[5,10]$ and other is due to hike in monetary cost of electricity [13]. The manual faults can be resolved by using monitoring and virtualization [12]. Second problem can be resolved by harnessing the renewable energy [9]. The two levels in energy consumption problem are shown in Figure 2

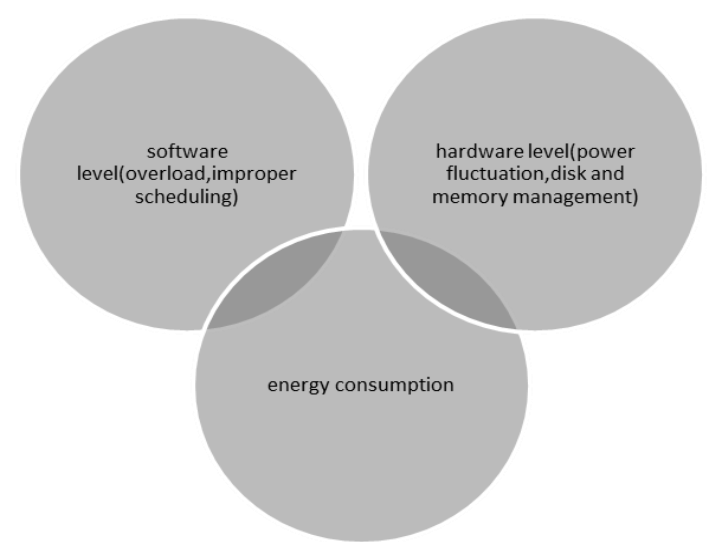

Figure2. Levels in energy consumption

\subsubsection{Monitoring}

It revealed that you cannot improve what you do not measure. Power metering is essential to construct power models that knowledge the system to know the energy consumed by a particular device, and how it can be reduced [12]. The virtual machine power metering follows the three steps such as: information collection, modelling and estimation in a while the unified efficiency of a data centers are measured and improve its performance per-watt.

\subsubsection{Virtualization}

Virtualization technology provides the flexible resource provisioning and migration of machine state [5]. Due to hot spot, excess space capacity and load imbalance which migrates the machine. Virtualization enables the consolidation, load balancing and hot spot mitigation [4]. It allocate data center resources dynamically based on application demands and support green computing by optimizing number of servers in use. 


\subsubsection{Renewable energy}

Cloud computing uses the brown energy which leads to hike in electricity bill and increase energy consumption. Harnessing renewable energy is used to address many issues like carbon emission, greenhouse gas emission and energy consumption [8], [9]. Renewable energy sources have less carbon emission rate rather than fossil fuels.

\section{EXISTING ENERGY EFFICIENT MODELS IN THE CLOUD 3.1. Workload Consolidation}

Srikantaiah et al. [22] have analyzed the relationship between resource and energy consumption. Moreover, the performances of workload consolidation were evaluated. The authors used two main features including, disk usage and CPU cycles in a bin-packing problem for task consolidation. Based on Pareto frontier algorithm the authors merged tasks and balance energy consumption by computing optimal points. The proposed technique was to compute optimal points from profiling data. An energy aware resource allocation mechanism used profiling step. The Euclidean distance measure between the current and the ideal point for each server is computed by profiling step.

\subsection{Virtual Machine Power Metering}

ChonglinGu et al. [12] have proposed virtual machine power metering which reduces the power consumption of data centers. There are three steps in virtual power metering such as information collection, modeling, and estimation. Power measured for the servers by deploying external meter and internal meter. It applies two methods as white box method and black box method. The black box method is used widely. The power meter is needed for virtual machine service billing, power budgeting, power saving scheduling. The unified efficiency of a data centers are measured and improve its performance per-watt.

\subsection{Energy Conservation Techniques}

Mehiar Dabbagh et al. [22] have analysed power management techniques that exploit virtualization technology to save energy. Prediction, consolidation and over commitment techniques are deployed. Workload prediction is used to turn off the unused physical machines to save energy. The overloaded machines are consolidated by virtual machine placement and workload consolidation. Resource over commitment is to reserve the resources in overly manner. It achieves the green energy.

\subsection{Virtual Machine Scheduling}

Dong Jiankang et al. [20] leveraged virtual machine scheduling to solve the combination of bin packing problem and quadratic assignment problem. It employs the two stage heuristic algorithm with virtual machine placement and migration. Virtual machine placement is to meet the physical capacity and network bandwidth. Virtual machine migration to minimize the migration costs to optimize the network maximum link utilization to reduce the energy consumption. $\mathrm{c}++$ is used to develop the algorithm. Compared to random algorithm the energy consumption is low.

\subsection{Renewable energy - aware migration}

UttamMandal et al. [8] have developed the renewable energy aware cloud service and virtual machine migration to relocate energy demand using resource allocation technique. This technique 
is used to migrate from one data center to another if more renewable energy is available in destination. A virtual machine is migrated to more green energy powered data center. Renewable energy aware migration tries to replace brown energy with green energy.

\subsection{Tasks Oriented Energy-Aware Scheduling}

Xiaomin Zhu et al. [15] have proposed energy aware scheduling and rolling horizon is named as EARH algorithm. Algorithm employs a rolling horizon optimization policy integrated with energy aware scheduling. Rolling horizon has a real time controller and virtual machine controller to hold new task along with the waiting task. Rolling horizon adjusts the tasks. After that the energy aware scheduling algorithm performs the resource scale up and scale down. It was implemented by cloudsim toolkit. The experimental results indicate that scheduling quality is improved and it saves the energy

\subsection{Ant Colony System}

Fahimeh Farahnakian et al. [19] have proposed ant colony based virtual machine consolidation which uses artificial ants to consolidate virtual machine into a reduced number of physical machines based on the current resource requests and ants work in parallel to build virtual machine migration. It composed with global and local agent. Global agent consolidates virtual machine into reduced number of physical machine. Local agent detects physical machine status. It was implemented by cloudsim toolkit. Result shows that this technique reduces the energy consumption up to $53.4 \%$ than the dynamic virtual machine consolidation.

\subsection{Dynamic Resource Allocation}

Zhen Xiao et al. [4] have proposed virtualization technology. Virtualization enables the consolidation, load balancing and hot spot mitigation. Virtualization technology provides the flexible resource provisioning and migration of machine state. Due to hot spot, excess space capacity and load imbalance which migrates the machine. The SKEWNESSS algorithm is used to measure the uneven utilization of server. Algorithm is simulated by trace driven simulation. It achieves green computing.

\subsection{Energy-Aware Scheduling}

Li Hongyou et al. [6] have marshalled the workload aware consolidation technique. Energy-aware Scheduling algorithm and the Energy- aware Live Migration algorithms are used. It accords the users to access the multiple resources concurrently in cloud data center. Algorithms audit the problem of consolidating heterogeneous workloads. Simulation results show that both algorithms convince the promising energy saving capability.

\subsection{Energy-Aware Task Consolidation}

Ching-Hsien Hsu et al. [11] have developed a technique that reduces energy consumption is energy-aware task consolidation (ETC) technique. ETC restricts CPU use below a specified peak threshold. Task consolidation is the major work of ETC. When a task migrates to other virtual clusters considered as network latency by energy cost model. Compared ETC with MAXUTIL for evaluation. MAXUTIL is a greedy algorithm that aspires to maximize cloud computing resources. The simulation result shows that 17\% improvement over MAXUTIL. 
International Journal of UbiComp (IJU), Vol.6, No.3, July 2015

\begin{tabular}{|c|c|c|c|}
\hline $\begin{array}{l}\text { Techniques and } \\
\text { Algorithms }\end{array}$ & Hardware / Datasets & Tools & Parameter Analysis \\
\hline $\begin{array}{l}\text { Energy Conservation } \\
\text { Techniques }\end{array}$ & $\begin{array}{l}\text { No specific environmental } \\
\text { set up. }\end{array}$ & Map reduce & $\begin{array}{l}\text { CPU utilization } \\
\text { Memory utilization }\end{array}$ \\
\hline $\begin{array}{l}\text { Virtual Machine Power } \\
\text { Metering }\end{array}$ & $\begin{array}{l}\text { A Power Edge blade } \\
\text { servers with Intel E5620 } \\
\text { CPU and } 24 \text { GB of RAM. }\end{array}$ & $\begin{array}{l}\text { Oprofile, pfmon, } \\
\text { perf-suite }\end{array}$ & Error rate \\
\hline Workload Consolidation & Data center data sets & maple & CPU cycles disk usage \\
\hline $\begin{array}{l}\text { Virtualization technology, } \\
\text { Concept of SKEWNESS. }\end{array}$ & $\begin{array}{l}\text { A group of } 30 \text { Dell Power } \\
\text { Edge blade servers with } \\
\text { Intel E5620 CPU and } 24 \\
\text { GB of RAM. The server } \\
\text { runs on Xen-3.3 and Linux } \\
2.6 .18\end{array}$ & Perfmon & Watt-meter \\
\hline $\begin{array}{l}\text { Renewable energy - } \\
\text { aware migration }\end{array}$ & Not mentioned & Not mentioned & Not mentioned \\
\hline $\begin{array}{l}\text { Energy aware task } \\
\text { consolidation(ETC) }\end{array}$ & Data center data sets & Cloud sim toolkit & $\begin{array}{l}\text { Number of nodes } \\
\text { Workloads }\end{array}$ \\
\hline $\begin{array}{l}\text { Consolidation of virtual } \\
\text { machine }\end{array}$ & Data center data sets & $\begin{array}{l}\text { Ad-hoc } \\
\text { simulator }\end{array}$ & $\begin{array}{l}\text { Number of active } \\
\text { servers, } \\
\text { Migrations per hour, } \\
\text { power }\end{array}$ \\
\hline $\begin{array}{l}\text { EARH scheduling } \\
\text { algorithm }\end{array}$ & $\begin{array}{l}\text { No specific environmental } \\
\text { set up. }\end{array}$ & $\begin{array}{l}\text { Cloud sim } \\
\text { Toolkit }\end{array}$ & $\begin{array}{l}\text { Task count, } \\
\text { Task arrival rate, } \\
\text { Task deadline }\end{array}$ \\
\hline $\begin{array}{l}\text { Dynamic consolidation of } \\
\text { virtual machines }\end{array}$ & Hadoop testbed & Cloudsim toolkit & $\begin{array}{l}\text { Energy consumption, } \\
\text { Number of virtual } \\
\text { machine migration }\end{array}$ \\
\hline $\begin{array}{l}\text { Virtual machine } \\
\text { scheduling algorithm } \\
\text {. }\end{array}$ & $\begin{array}{l}\text { No specific environmental } \\
\text { set up. }\end{array}$ & $\mathrm{C}++$ & $\begin{array}{l}\text { Energy consumption, } \\
\text { Total communication } \\
\text { Traffic, } \\
\text { Maximum link } \\
\text { utilization }\end{array}$ \\
\hline
\end{tabular}

Table 1. Comparison of the existing methods

\section{CONCLUSIONS}

In this survey, we focused on key parameters of energy consumption problem. The existing models are explained. From the survey it is found that some of the models are yet to be implemented. The power metering and virtualization technologies are useful to utilize the energy in efficient way. Cloud computing much more energy efficient, there are still many technological solutions and enhanced green cloud framework is needed to achieve energy efficient cloud computing in reality. 


\section{REFERENCES}

[1] Bernadette Addis, DaniloArdagna, Barbara Panicucci, Mark S. Squillante and Li Zhang, "A Hierarchical Approach for the Resource Management of Very Large Cloud Platforms," IEEE Transactions on Dependable and Secure Computing, vol. 10, no. 5, September/October 2013.

[2] Federico Larumbe and Brunilde Sanso," A Tabu Search Algorithm For The Location Of Data Centers And Software Components In Green Cloud Computing Networks", IEEE Transactions On Cloud Computing, Vol. 1, No. 1, January-June 2013.

[3] Carlo Mastroianni, MichelaMeo and Giuseppe Papuzzo,'Dynamic Heterogeneity-Aware Resource Provisioning In The Cloud", IEEE Transactions On Cloud Computing, Vol. 1, No. 2, JulyDecember 2013.

[4] Zhen Xiao, Weijia Song, and Qi Chen, " Dynamic Resource Allocation Using Virtual Machines for Cloud Computing Environment," IEEE Transactions on Parallel and Distributed Systems, vol. 24, no. 6, June 2013.

[5] Mayank Mishra, Anwesha Das, Purushottam Kulkarni, and Anirudha Sahoo," Dynamic Resource Management Using Virtual Machine Migrations," IEEE Communications Magazine, 01636804/12, September 2012.

[6] Li Hongyou, Wang Jiangyong, Peng Jia, Wang Junfeng, Liu Tang, "Energy-Aware Scheduling Scheme Using Workload-Aware Consolidation Technique In Cloud Data Centres", China Communications, December 2014.

[7] Michael Cardosa, Aameek Singh, Himabindu Pucha and Abhishek Chandra," Exploiting SpatioTemporal Trade-offs For Energy-Aware Map reduce In The Cloud", IEEE Transactions On Computers, Vol. 61, No. 12, December 2012.

[8] UttamMandal, M.FarhanHabib, Shuqiang Zhang and Biswanath Mukherjee, Davis Massimo Tornatore, Davis and Politecnico Di Milano" Greening the Cloud Using Renewable-EnergyAware Service Migration", IEEE Network, November/December 2013.

[9] Wei Deng, Fangming Liu and Hai Jin," Harnessing Renewable Energy In cloud Datacentres Opportunities and Challenges", IEEE Network, January/February 2014.

[10] Konstantinos Tsakalozos, Mema Roussopoulos, and Alex Delis," Hint-Based Execution of Workloads In Clouds With Nefeli”, IEEE Transactions On Parallel And Distributed Systems, Vol. 24, No. 7, July 2013.

[11] Ching-Hsien Hsu, KennD.Slagter, Shih-Chang Chen, Yeh -Chinh Chung," Optimizing Energy Consumption with Task Consolidation in Cloud", Information Sciences, No. 3, March 2014.

[12] Chonglin Gu, Hejiao Huang, and Xiaohua Jia,” Power Metering For Virtual Machine In Cloud Computing - Challenges And Opportunities", IEEE Access, Vol. 2, Sep 2014.

[13] Jianguo Yao, Xue Liu, and Chen Zhang," Predictive Electricity Cost Minimization Through Energy Buffering In Data Centers”, IEEE Transactions On Smart grid, Vol.5,No.1,January2014.

[14] Carlo Mastroianni, MichelaMeo, and Giuseppe Papuzzo," Probabilistic Consolidation Of Virtual Machines In Self-Organizing Cloud Data Centers", IEEE Transactions On Cloud Computing, Vol. 1, No. 2, July-December 2013.

[15] Xiaomin Zhu, Laurence T. Yang, Huangke Chen, Ji Wang, Shu Yin and Xiaocheng Liu," RealTime Tasks Oriented Energy-Aware Scheduling In Virtualized Clouds", IEEE Transactions On Cloud Computing, Vol. 2/April-June 2014.

[16] JianyingLuo, Lei Rao, and Xue Li," Temporal Load Balancing With Service Delay Guarantees For Data Center Energy Cost Optimization", IEEE Transactions On Parallel And Distributed Systems, Vol. 25, March 2014. 
[17] Mehiar Dabbagh, Bechir Hamdaoui, Mohsen Guizani, and Ammar Rayes,” Toward EnergyEfficient Cloud Computing Prediction, Consolidation, And Over commitment", IEEE Network, March/April 2015.

[18] Weiwen Zhang, Yonggang Wen, and Hsiao-Hwa Chen," Toward Transcoding as a Service Energy-Efficient Offloading Policy for Green Mobile Cloud", IEEE Network, November/December 2014.

[19] Fahimeh Farahnakian, Adnan Ashraf, Tapio Pahikkala, Pasi Liljeberg, Juha Plosila, Ivan Porres, And Hannu Tenhunen,"Using Ant Colony System To Consolidate Vms For Green Cloud Computing", IEEE Transactions On Services Computing, Vol. 8, No. 2, March/April 2015.

[20] Dong Jiankang, Wang Hongbo, Li Yang yang, Cheng Shiduan,"Virtual Machine Scheduling For Improving Energy Efficiency In Iaas Cloud", China Communications , March 2014.

[21] Elizabeth Sylvester Mkoba, Mokhtar Abdullah Abdo Saif," A Survey On Energy Efficient With Task Consolidation In The Virtualized Cloud Computing Environment ", IJRET: International Journal of Research in Engineering and Technology.

[22] Srikantaiah S, Kansal A, Zhao F (2008)," Energy aware consolidation for cloud computing." In: Conference on power aware computer and systems (HotPower '08)

\section{Authors}

P.MALATHI received the B.E degree in computer science and engineering from Nandha Engineering College in 2014. She is currently doing her M.E Computer science and Engineering in Nandha engineering college, Erode, India.

DR.S.ARUMUGAM,completed B.E and M.Sc.,(Engg.),, from P.S.G College of Technology,University of Madras in the year 1971 and 1973 respectively.he completed his Ph.D from College of Engineering, Guindy,Anna University in the year 1990. He worked in the Department of Technical Education, Government of Tamilnadu from the year 1974 to 2007 at various capacities,AssociateLecturer,Lecturer,Assistant Professor,Professor,Principal and Additional Director Of Technical Education. He is presently working as professor and CEO in Nandha engineering college. He has

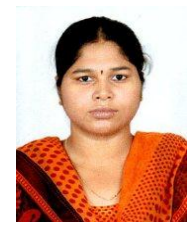
published more than 100 papers in various journals. He had a profession membership in ISTE, CSI, IEEE, IE (I) and IETE. 22 faculty members have completed Ph.D. under his guidance and currently he is supervising 20 research scholars. 